\title{
Rotating compact stars with exotic matter
}

\author{
Sarmistha Banik ${ }^{(a)}$, Matthias Hanauske ${ }^{(\mathrm{b})}$, Debades Bandyopadhyay ${ }^{(\mathrm{a})}$ and Walter \\ Greiner $^{(\mathrm{c})}$ \\ (a) Saha Institute of Nuclear Physics, 1/AF Bidhannagar, Calcutta 700 064, India \\ (b) Institut für Theoretische Physik, J. W. Goethe Universität, D-60054 Frankfurt am Main, \\ Germany \\ (c) Frankfurt Institute for Advanced Studies (FIAS), J. W. Goethe Universität, D-60054 Frankfurt \\ am Main, Germany
}

\begin{abstract}
We have constructed models of uniformly rotating compact stars including hyperons, Bose-Einstein condensates of antikaons and quarks. First order phase transitions from hadronic to antikaon condensed matter and then to quark matter are considered here. For the equation of state undergoing phase transitions to antikaon condensates, the third family of compact stars are found to exist in the fixed angular velocity sequences. However, the third family solution disappears when the compact stars rotate very fast. For this equation of state, the fixed baryon number supramassive sequence shows a second stable part after the unstable region but no back bending phenomenon. On the other hand, we observe that the rotation gives rise to a second maximum beyond the neutron star maximum for the equation of state involving phase transitions to both antikaon condensed and quark matter. In this case, the back bending phenomenon has been observed in the supramassive sequence as a consequence of the first order phase transition from $K^{-}$condensed to quark matter. And the back bending segment contains stable configurations of neutron stars.
\end{abstract}

PACS: 26.60.+c, 21.65.+f, 97.60.Jd, 95.30.Cq

\section{INTRODUCTION}

The study of dense and cold matter in neutron star interior has gained momentum after the advances in observational astronomy. One important goal of various observations is to measure masses and radii of compact stars [1-4]. This might, in turn, constrain the composition and equation of state (EoS) of dense matter in compact stars. The composition and structure of a neutron star depend on the nature of strong interaction. In neutron star cores, the matter density could exceed by a few times normal nuclear matter density. Since the baryon and lepton chemical potentials increase rapidly with density in the core, different exotic forms of matter with a large strangeness fraction such as hyperon matter [5], Bose-Einstein condensates of strange mesons [6-14] and quark matter $[5,7,15,16]$ may appear there. 
Neutron star matter including exotic phases was studied extensively using relativistic field theoretical models [17] and chiral models [18]. These studies have revealed many important aspects of the EoS of dense matter and static neutron star properties. It was noted that the appearance of exotic forms of matter in the high density regime resulted in kinks in the EoS $[12,19,20]$. As a result, there was a discontinuity in the speed of sound. It has a great implication. There is a growing interest to understand whether a stable sequence of compact stars could exist in nature beyond a white dwarf and neutron star branch. Gerlach [21] first showed that it could be a possibility if there was a jump in an EoS or discontinuity in the speed of sound. Later various groups constructed equations of state undergoing first order phase transitions from hadronic to quark matter [20,22], hadronic to antikaon condensed matter [12] and hadronic to hyperonic matter [19] which gave rise to a stable branch of non rotating compact stars called the third family beyond the neutron star branch. It was noted that nonidentical stars of same mass but different radii and compositions could exist because of partial overlapping mass regions of two branches. These pairs are called neutron star twins.

On the other hand, the properties of rapidly rotating neutron stars may also constrain the EoS of dense matter. In particular, the maximum angular velocity of rotating neutron stars is quite sensitive to the EoS [23,24]. An EoS predicting smaller Keplerian frequencies than the observed frequencies is not allowed. Also, it was earlier predicted that the back bending phenomenon in a fixed baryon number evolutionary sequence could shed light on the existence of quark matter in the neutron star interior $[25,26]$.

In this paper, we are interested to investigate the impact of exotic forms of matter on various properties of rotating compact stars. In this respect, we exploit equations of state including hyperons, Bose-Einstein condensates of strange mesons and quarks and derived them from the relativistic field theoretical model and chiral model. The paper is organised in the following way. In Section II, we discuss the space-time geometry of stationary and axisymmetric rotating stars and their stability briefly. We describe equations of state adopted in this calculation in Section III. In Section IV, we discuss results of our calculation. Section $\mathrm{V}$ is devoted to summary and conclusions.

\section{UNIFORMLY ROTATING NEUTRON STARS}

Models of rotating neutron stars were developed by various groups [27-36]. Here we consider stationary and axisymmetric equilibrium configurations of rotating neutron stars. In this case, the metric has the form [35]

$$
d s^{2}=-e^{\gamma+\rho} d t^{2}+e^{2 \alpha}\left(d r^{2}+r^{2} d \theta^{2}\right)+e^{\gamma-\rho} r^{2} \sin ^{2} \theta(d \phi-\omega d t)^{2},
$$

where metric potentials $\gamma, \rho$ and $\alpha$ and $\omega$ depend on radial coordinate $r$ and polar angle $\theta$. The compact star matter is considered to be a perfect fluid and the energy density and pressure are determined from the stress-energy tensor. Here we construct models of uniformly rotating neutron star using a code based on Komatsu-Eriguchi-Hachisu method $[32,33]$ and written by Steirgioulas [37] for three equations of state. In this paper, we study both the static and mass shedding or Keplerian limit which is attained by a stable neutron star rotating with a maximum frequency called the Kepler frequency $\Omega_{K}$ before the mass 
loss at the equator occurs [35]. Also, we focus our attention on rotating neutron stars along evolutionary sequences where the total baryon number of a star is conserved. There are two kinds of evolutionary sequences - normal and supramassive. Normal evolutionary sequence has one end connecting the static limit whereas the supramassive evolutionary sequence does not contain any static solution. Along an evolutionary sequence, the stability condition is given by $[34,35]$

$$
\frac{d J}{\left.d \epsilon_{c}\right|_{N_{b}=\text { const. }}}<0,
$$

where $J$ is the angular momentum, $\epsilon_{c}$ is the central energy density and $N_{b}$ is the total baryon number.

\section{EQUATIONS OF STATE}

In this paper, we use three equations of state constructed by us. One of these EoS is constructed from a QCD-motivated hadronic chiral $\mathrm{SU}(3)_{L} \times \mathrm{SU}(3)_{R}$ model [18]. In this model, the Lagrangian is constructed with respect to the nonlinear realization of chiral $\mathrm{SU}(3)_{L} \times \mathrm{SU}(3)_{R}$ symmetry; masses of heavy baryons and mesons are generated by spontaneous symmetry breaking and masses of the pseudoscalar bosons are the results of explicit symmetry breaking. A dilaton field is introduced to describe the gluon condensate. This EoS does not involve any phase transition and describes matter involving only neutrons, protons, hyperons and leptons. It is denoted as CHM case. This EoS was already used to study neutron star properties [18,38].

Next, we discuss the EoS undergoing a first order phase transition from hadronic to $K^{-}$condensed matter and then a second order $\bar{K}^{0}$ condensation [12]. This is hereafter denoted as HK case. In the last case, the EoS involves first order phase transitions from nuclear to $K^{-}$condensed matter and then from $K^{-}$condensed matter to quark matter. It is referred to as HKQ case. In both cases, we adopt a relativistic field theoretical model to describe the pure hadronic and antikaon condensed matter and their mixed phase $[9,12]$. The constituents of the hadronic phase for HK case are the members of the baryon octet and leptons. On the other hand, the pure $\bar{K}$ condensed phase consists of members of the baryon octet, $K^{-}, \bar{K}^{0}$ mesons and leptons for HK case. Similarly, the compositions of the hadronic and antikaon condensed phases for HKQ case are $n, p$, leptons and $n, p, K^{-}$mesons and leptons respectively. In the condensed phase, baryons are embedded in the condensates. The baryon-baryon and baryon-(anti)kaon interactions are mediated by the scalar and vector mesons. The model is also extended to include hyperon-hyperon interaction [8]. On the other hand, the pure quark matter is composed of $u, d, s$ quarks and electrons. The quark phase is described by the MIT bag model. Each pure phase maintains local charge neutrality and $\beta$-equilibrium conditions. The mixed phase of any two pure phases is described by the Gibbs phase rules along with global charge neutrality and total baryon number conservation. The meson-baryon and meson-kaon coupling constants used in our calculations for HK and HKQ cases are recorded in Ref. [12]. An antikaon optical potential depth of $U_{\bar{K}}\left(n_{0}\right)=-160 \mathrm{MeV}$ at normal nuclear matter density is used in the calculation of EoS for HK and HKQ cases.

Also, we use a bag constant $B^{1 / 4}=200 \mathrm{MeV}$ and strange quark mass $m_{s}=150 \mathrm{MeV}$ for the quark matter. 
The structure of static compact stars was already investigated for HK case using TolmanOppenheimer-Volkoff (TOV) equations [12]. For HK case, it was noted that the threshold of $K^{-}$condensation was very sensitive to the antikaon optical potential depth $\left(U_{\bar{K}}\left(n_{0}\right)\right)$ at normal nuclear matter density. We performed this calculation for a set of antikaon optical potential depths from -100 to $-180 \mathrm{MeV}$. For $\left|U_{\bar{K}}\left(n_{0}\right)\right|<160 \mathrm{MeV}$, the early appearance of negatively charged hyperons did not allow $K^{-}$condensate to appear in the system. However, $K^{-}$condensate appeared before the formation of negatively charged $\Xi$ hyperons for $\left|U_{\bar{K}}\left(n_{0}\right)\right| \geq 160 \mathrm{MeV}$. So we performed the static structure calculation for HK case using $U_{\bar{K}}\left(n_{0}\right)=-160$ and $-180 \mathrm{MeV}$. In this calculation, we found a new family of compact stars called the third family beyond the neutron star branch [12]. After the maximum mass star in the neutron star branch, there is an unstable region followed by a stable third family branch of superdense stars. However, we did not find this third family branch when we performed calculation with $U_{\bar{K}}\left(n_{0}\right)=-180 \mathrm{MeV}$. So the third family branch of compact stars appears in a certain range of antikaon optical potential depth.

\section{RESULTS AND DISCUSSION}

Now we present our results for CHM, HK and HKQ cases. In Fig. 1, equations of state (pressure versus energy density) are displayed for those cases. For HK and HKQ cases, the mixed phase of hadronic and $K^{-}$condensed matter begins at energy density $\epsilon=6.1132 \times 10^{14}$ $\mathrm{g} / \mathrm{cm}^{3}$ and ends at $\epsilon=1.1651 \times 10^{15} \mathrm{~g} / \mathrm{cm}^{3}$. In HK case, $\Lambda$ hyperons appear in the mixed phase and $\bar{K}^{0}$ condensation sets in just after the mixed phase is over [12]. Also, heavier hyperons are populated at higher baryon density for HK case [12]. On the other hand, the first order phase transition from $K^{-}$condensed matter to quark matter for HKQ case starts at $\epsilon=1.5884 \times 10^{15} \mathrm{~g} / \mathrm{cm}^{3}$ and terminates at $\epsilon=2.7688 \times 10^{15} \mathrm{~g} / \mathrm{cm}^{3}$. The overall EoS for HK case is softer than that of HKQ case whereas that of CHM model is stiffer than HK case but softer compared with HKQ case.

The gravitational mass for static neutron star sequence and the sequence of neutron stars rotating at their respective Kepler frequencies are plotted with central energy density in Fig. 2 for CHM, HK and HKQ cases. The bottom three curves represent the static limit sequences calculated using TOV equations and the top three curves correspond to the mass shedding limit sequences for the above mentioned EoS. We have already mentioned that the EoS for HK case gives rise to the third family of superdense stars beyond the neutron star branch. The maximum mass stars on the neutron star branch and the third family branch are indicated by solid and open circles respectively. The maximum gravitational masses, equatorial radii and the corresponding central energy densities for the static and mass shedding limit are given by Table 1 . Also, the Kepler periods for maximum masses in mass shedding limit sequences are recorded in the Table. For HK case, the second row gives the relevant quantities for the third family branch in the static sequence. The compact star in the third family has a smaller radius than the neutron star as it is evident from the Table. With the help of the central energy densities corresponding to maximum mass static and rotating stars for HK and HKQ case from Table 1 and Fig. 1, we find that the corresponding central pressures for HK case are smaller than that of HKQ case. Therefore, the overall EoS from the center to the surface of a maximum mass star is softer in HK case compared with that of HKQ case. This leads to smaller maximum masses in the static and mass shedding 
limit sequence for HK case than that of HKQ case.

The gravitational mass as a function of equatorial radius is shown in Fig. 3a, Fig. 3b and Fig. 3c for CHM, HK and HKQ cases respectively. Also, we have plotted fixed angular velocity sequences in those figures. Each fixed angular velocity curve is denoted by a value of $\Omega$. In Fig. 3a, fixed baryon number curve I with $N_{b}=1.71 \times 10^{57}$ and curve II with $N_{b}=2.18 \times 10^{57}$ stand for normal sequences and curve III with $N_{b}=2.51 \times 10^{57}$ is the supramassive sequence. In Fig. $3 \mathrm{~b}$ and $3 \mathrm{c}$, fixed baryon number curves I and II with $N_{b}=1.94 \times 10^{57}\left(2.15 \times 10^{57}\right)$ and $2.06 \times 10^{57}\left(2.34 \times 10^{57}\right)$ are normal sequences and curves III and IV with $N_{b}=2.09 \times 10^{57}\left(2.45 \times 10^{57}\right)$ and $2.50 \times 10^{57}\left(2.57 \times 10^{57}\right)$ are supramassive sequences for $\mathrm{HK}$ (HKQ) case respectively. In each figure, the solid circle exhibits the location of the maximum mass star in the neutron star branch. We note that there are some interesting structures in the fixed angular velocity curves for HK and HKQ cases which are undergoing first order phase transitions from hadronic to antikaon condensed and then to quark matter.

For HK case, the static sequence represented by the bold line at the bottom in Fig. 3b has some interesting structure. After the maximum neutron star mass, there is an unstable region followed by a stable third family branch of compact stars [12]. The stellar mass peak in the third family branch is denoted by an open circle. So far some EoS undergoing first order phase transitions from hadronic to hyperon matter [19], antikaon condensed matter [12] and quark matter [16,20,22] gave rise to the stable third family branch beyond the neutron star branch in the static case. Generally, it was noted that if the neutron star maximum occurred in the mixed phase or just after that, there would be a third family branch because of the jump in the EoS [21].

Now the question is whether the third family branch could still exist under the influence of rotation. In Fig. 3b, we find that the third family branch exists for the fixed angular velocity sequence with $\Omega=2158 \mathrm{~s}^{-1}$. But there is no third family solution for fixed angular velocity curves with $\Omega=3000 \mathrm{~s}^{-1}, \Omega=4900 \mathrm{~s}^{-1}$ and beyond. The disappearance of the third family branch may be understood in the following way. It was argued that the third family solution was very sensitive to the behaviour of EoS at higher density [22]. The rotating stars have smaller central energy densities than the non rotating stars with the same gravitational mass. This is attributed to the effect of the centrifugal force. It is also evident from the higher angular velocity and mass shedding limit curves that the positions of maximum star masses move towards smaller central energy densities. Consequently, the third family branch disappears because the high density part of the EoS which is responsible for the third family solution, can not be probed in fast rotating compact stars.

On the other hand, there is no third family branch in the static limit sequence for HKQ case in Fig. 3c. As angular velocity increases, the curves become more and more flattened due to the softening in the EoS by the antikaon condensate and quarks. This leads to a local minimum as it is evident from fixed angular velocity sequences with $\Omega=5500 \mathrm{~s}^{-1}$ and $\Omega=6100 \mathrm{~s}^{-1}$ in Fig. 3c. It has been noted in Ref.[39] that this local minimum in M-R curve at a fixed angular velocity is "strictly" connected to the back bending phenomenon. We also find such a connection in this case and discuss it later. The curves for $\Omega=5500 \mathrm{~s}^{-1}$ and $\Omega=6100 \mathrm{~s}^{-1}$ in Fig. 3c exhibit two local maxima and they are denoted by solid circles. Here the parts of fixed angular velocity curves where compact star masses decrease as a function of radius or central energy density between two local maxima, are not unstable. This may 
be understood by studying the stability of fixed baryon number sequences. For example, it will be evident from the stability analysis of the fixed baryon number curve HKQ, III which touches the fixed angular velocity curve with $\Omega=5500 \mathrm{~s}^{-1}$ around the local minimum. We discuss this stability analysis in the following paragraph. It is noted here that the backward turning portions of curves III and IV in Fig. 3c are actually unstable parts.

In Fig. 4, we have shown the stability (angular momentum versus central energy density) of fixed baryon number sequences for HK and HKQ cases. The curve for HK case corresponds to the baryon number $N_{b}=2.09 \times 10^{57}$ whereas it is $N_{b}=2.45 \times 10^{57}$ for HKQ case. These fixed baryon number sequences for HK and HKQ cases are shown by curve III in Fig. 3b and in Fig. 3c respectively. In Fig. 4, we note that angular momentum, $J$, initially decreases with central energy density for both cases. This is shown by solid lines. It implies that the fixed baryon number sequences are stable in these regions as demanded by Eqn.(2). After the stable parts, there are unstable regions where angular momentum increases with central energy density. The unstable parts are represented by dotted lines. From the stability analysis of HKQ, III curve, we find that it is the stable part of the curve which touches the fixed angular velocity sequence with $\Omega=5500 \mathrm{~s}^{-1}$ around the local minimum in Fig. 3c. On the other hand, we find an interesting structure in HK, III curve. After the unstable part in HK, III curve, there is a stable region followed by an unstable region. This second stable part in HK case is the outcome of the high density behaviour of the EoS which actually gives rise to the third family of compact stars in Fig. 2 and Fig. 3b.

A rotating neutron star slowly loses its energy and angular momentum through electromagnetic and gravitational radiation with time. However, it conserves the total baryon number during this evolution. Therefore, fixed baryon number sequences or evolutionary sequences provide valuable informations about isolated rotating stars. In the following paragraphs, we analyse normal and supramassive sequences of Figures 3. We show the behaviour of angular velocity with angular momentum for CHM, HK and HKQ cases in Fig. 5a, Fig. 5b and Fig. 5c respectively. The mass shedding limit sequence in each figure is shown by a light solid line. Also we have plotted normal and supramassive sequences in these figures. The stable parts of various sequences are displayed by dark solid lines and the unstable parts by dotted lines. It is worth mentioning here that the stability of each curve is determined by Eqn. (2). For CHM case, we have shown two normal sequences by curves I and II and a supramassive sequence by curve III. We find that neutron stars along normal sequences spin down as they lose angular momentum. However, the compact star along the supramassive sequence spins up with angular momentum loss. This was already noted by various groups $[35,37]$. Similarly we plot normal and supramassive sequences for HK and HKQ cases in Fig. $5 \mathrm{~b}$ and Fig. 5c. The most interesting result is obtained from curve II of Fig. 5b. In this case, we have an unstable part followed by another stable part. The stable region beyond the unstable part may be attributed to the high density behaviour of EoS for HK case. Already we have discussed that this EoS gives rise to a stable third family branch of compact stars beyond the neutron star branch in the static limit and fixed angular velocity sequences. In Fig. 5b, we also note that the compact star on the second stable branch rotates faster.

Now we display moment inertia (I) versus angular velocity $(\Omega)$ for HK and HKQ cases in Fig. 6a and Fig. 6b respectively. In both figures, normal sequence (curve I) and supramassive sequences (curves III and IV) are displayed. In Fig. 6a and Fig. 6b, we find that the moment of inertia always diminishes with decreasing angular velocity for normal sequences. 
In Fig. 6a, supramassive sequences have both stable and unstable parts denoted by solid and dotted lines respectively. For curve III of Fig. 6a, the moment of inertia initially decreases as the star rotates slowly along the stable part of the sequence. But the compact star spins up just before the beginning of the unstable part as it has been already observed for curve III of Fig. 5b. After the unstable region, there is a stable part followed by an unstable part in curve III of Fig. 6a. The neutron star in the second stable part spins up with the loss of moment of inertia. On the other hand, the neutron star always spins up along the stable part of curve IV in Fig. 6a.

In Fig. 6b, the supramassive sequence denoted by curve III exhibits an interesting structure. After the initial spin down of the neutron star along this sequence, there is a spin up followed by another spin down. This is known as back bending phenomenon. We observe that this back bending phenomenon is definitely connected to the appearance of the local minimum in the fixed angular velocity sequences in Fig. 3c. For example, the fixed baryon number curve III of Fig. 6b actually passes through the local minimum of the fixed angular velocity curve with $\Omega=5500 \mathrm{~s}^{-1}$ of Fig. 3c. Such a feature was earlier observed for an EoS involving a strong first order hadron-quark phase transition [25] and for the EoS including hyperons degrees of freedom [39]. In our calculation, the EoS for HKQ case first undergoes the hadron-antikaon condensed matter phase transition and then the antikaon condensed-quark matter phase transition. It is the phase transition to quark matter which is responsible for the back bending in curve III of Fig. 6b. Though we have a first order hadron-antikaon condensed matter phase transition in HK case, we do not find any such event in this case as it is evident from the curves of Fig. 6a. It has been stressed that J versus $\Omega$ curve provides more insight into the back bending phenomenon than I versus $\Omega$ curve [39]. For the former curve tells us whether the back bending segment contains stable configurations or not. For HKQ case, the back bending phenomenon occurs in the stable part of the curve.

\section{SUMMARY AND CONCLUSIONS}

We have studied the effects of different forms of exotic matter such as hyperons, BoseEinstein condensates of antikaons and quarks on rotating neutron stars. For this calculation, we adopted three equations of state. The CHM case is described by a chiral model and this EoS does not involve any phase transition. On the other hand, the EoS for HK case involves a first order phase transition from hadronic to $K^{-}$condensed matter and then a second order $\bar{K}^{0}$ condensation whereas the EoS for HKQ case undergoes successive first order phase transitions from hadronic to $K^{-}$condensed matter and then to quark matter. In both cases, the hadronic phase is described by a relativistic field theoretical model.

We have calculated various properties of rotating neutron stars with those equations of state. The maximum gravitational mass in static and mass shedding limit sequences for HK case are smaller than that of HKQ case. For HK case, there exists a stable third family branch of compact stars beyond the neutron star branch in the static sequence.

Next we have investigated fixed angular velocity sequences for the above mentioned equations of state. With increasing rotation, the fixed angular velocity curves became more flattened due to softening in EoS. For HK case, we find that the third family branch disappears for larger angular velocities. On the other hand, for HKQ case, the flattening in 
the fixed angular velocity sequences exhibit minima. We have seen that these minima are actually connected to the back bending phenomenon.

We have further computed fixed baryon number normal and supramassive sequences. Using these evolutionary sequences, we have studied the stability and the behaviour of angular momentum and moment of inertia with angular velocity for different equations of state. For HK case, we observe that the supramassive sequence has a second stable segment followed by another unstable part. This second stable part is the result of the high density behaviour of the EoS for HK case which is responsible for the third family solution. It is found that neutron stars on the second stable branch rotate faster. For HKQ case, we observe the interesting back bending phenomenon in the moment of inertia versus angular velocity curve. And the back bending segment contains stable configurations of neutron stars. Phase transitions to antikaon condensed and quark matter in neutron star interior could be probed through the observation of back bending phenomenon.

\section{Acknowledgements}

This work is supported by the Department of Science and Technology (DST), India and German Academic Exchange Service (DAAD), Germany under a project based Personnel Exchange Programme (sanction order no. INT/DAAD/P-94/2003). 


\section{REFERENCES}

[1] J.A. Pons, F.M. Walter, J.M. Lattimer, M. Prakash, R. Neuhäuser and P. An, Astrophys. J 564, 981 (2002).

[2] J.J. Drake et al., Astrophysics J. 572, 996 (2002).

[3] F.M. Walter and J.M. Lattimer, Astrophys. J 576, L145 (2002).

[4] J. Cottam, F. Paerels and M. Mendez, Nature 420, 51 (2002);

C. Miller, Nature 420, 31 (2002).

[5] N. K. Glendenning, Compact stars, (Springer, New York, 1997).

[6] D.B. Kaplan and A.E. Nelson, Phys. Lett. B 175, 57 (1986);

A.E. Nelson and D.B. Kaplan, Phys. Lett. B 192, 193 (1987).

[7] M. Prakash, I. Bombaci, M. Prakash, Paul J. Ellis, J. M. Lattimer and R. Knorren, Phys. Rep. 280, 1 (1997).

[8] J. Schaffner and I.N. Mishustin, Phys. Rev. C 53, 1416 (1996).

[9] N.K. Glendenning and J. Schaffner-Bielich, Phys. Rev. Lett. 81, 4564 (1998).

[10] S. Pal, D. Bandyopadhyay and W. Greiner, Nucl. Phys. A674, 553 (2000).

[11] S. Banik and D. Bandyopadhyay, Phys. Rev. C 63, 035802 (2001).

[12] S. Banik and D. Bandyopadhyay, Phys. Rev. C 64, 055805 (2001).

[13] S. Banik and D. Bandyopadhyay, Phys. Rev. C 66, 065801 (2002).

[14] S. Banik and D. Bandyopadhyay, Phys. Rev. D 67, 123003 (2003).

[15] E. Farhi and R.L. Jaffe, Phys. Rev. D 30, 2379 (1984).

[16] K. Schertler, C. Greiner, J. Schaffner-Bielich and M.H. Thoma Nucl. Phys. A677, 463 (2000).

[17] J.D. Walecka, Theoretical Nuclear and Subnuclear Physics, (Oxford University Press, New York, 1995).

[18] M. Hanauske, D. Zschiesche, S. Pal, S. Schramm and H. Stöcker and W. Greiner, Astrophys. J. 537, 958, 2000.

[19] J. Schaffner-Bielich, M. Hanauske, H. Stöcker and W. Greiner, Phys. Rev. Lett. 89, 171101 (2002).

[20] I. N. Mishustin, M. Hanauske, A. Bhattacharyya, L. M. Satarov, H. Stöcker and W. Greiner, Phys. Lett. B 552, 1 (2003).

[21] U.H. Gerlach, Phys. Rev. 172, 1325 (1968).

[22] N. K. Glendenning and C. Kettner, Astron. Astrophys. 353, L9 (2000).

[23] T. Nozawa, M. Hashimoto, K. Oyamatsu and Y. Eriguchi, Phys. Rev. D 53, 1845 (1996).

[24] G.F. Burgio, H.-J. Schulze and F. Weber, Astron. Astrophys. 408, 675 (2003).

[25] F. Weber, Pulsars as Astrophysical Laboratories for Nuclear and Particle Physics, (Institute of Physics Publishing, Bristol and Philadelphia).

[26] N. K. Spyrou and N. Stergioulas, Astron. Astrophys. 395, 151 (2002).

[27] J. R. Wilson, Astrophys. J. 176, 195 (1972).

[28] S. Bonazzola and J. Schneider, Astrophys. J. 191, 273 (1974).

[29] E. M. Butterworth and J. R. Ipser, Astrophys. J. 204, 200 (1976).

[30] J. L. Friedman, J. R. Ipser and L. Parker, Astrophys. J. 304, 115 (1986).

[31] J. L. Friedman, J. R. Ipser and L. Parker, Phys. Rev. Lett. 62, 3015 (1989).

[32] H. Komatsu, Y. Eriguchi and I. Hachisu, MNRAS 237, 355 (1989).

[33] H. Komatsu, Y. Eriguchi and I. Hachisu, MNRAS 239, 153 (1989).

[34] G. B. Cook, S. L. Shapiro and S. A. Teukolsky, Astrophys. J. 398, 203 (1992). 
[35] G. B. Cook, S. L. Shapiro and S. A. Teukolsky, Astrophys. J. 422, 227 (1994).

[36] M. Salgado, S. Bonazzola, E. Gourgoulhon and P. Haensel, Astron. Astrophys. 291, 155 (1994).

[37] N. Stergioulas and J. L. Friedman, Astrophys. J. 444, 306 (1995).

[38] I. Shovkovy, M. Hanauske and M. Huang, Phys. Rev. D 67, 103004 (2003).

[39] J. L. Zdunik, P. Haensel, E. Gourgoulhon and M. Bejger, Astron. Astrophys. 416, 1013 (2004). 


\section{TABLES}

TABLE I. Maximum gravitational masses $\left(M_{G} / M_{\odot}\right)$, equatorial radii $(\mathrm{R})$ and their corresponding central energy densities $\left(\epsilon_{c}\right)$ for static $(P=0)$ and Keplerian limit $\left(\mathrm{P}=P_{K}=2 \pi / \Omega_{K}\right)$ with different EoS, where $P_{K}$ is the Kepler period in milliseconds. The second row for HK case denotes those of the third family branch for the static case.

\begin{tabular}{|lcccc|}
\hline \hline EoS & $P(m s)$ & $\epsilon_{c}\left(10^{15} \mathrm{~g} / \mathrm{cm}^{3}\right)$ & $M_{G} / M_{\odot}$ & \\
& & & & $R(\mathrm{~km})$ \\
\hline HK & 0 & 1.34 & 1.569 & 13.28 \\
& 0 & 2.69 & 1.553 & 10.98 \\
& 1.0276 & 1.04 & 1.944 & 19.09 \\
$\mathrm{HKQ}$ & 0 & & & 12.10 \\
& 0.8062 & 2.28 & 1.752 & 16.46 \\
$\mathrm{CHM}$ & 0 & 1.96 & 2.084 & 11.72 \\
& 0.8289 & 1.99 & 1.636 & 16.52
\end{tabular}




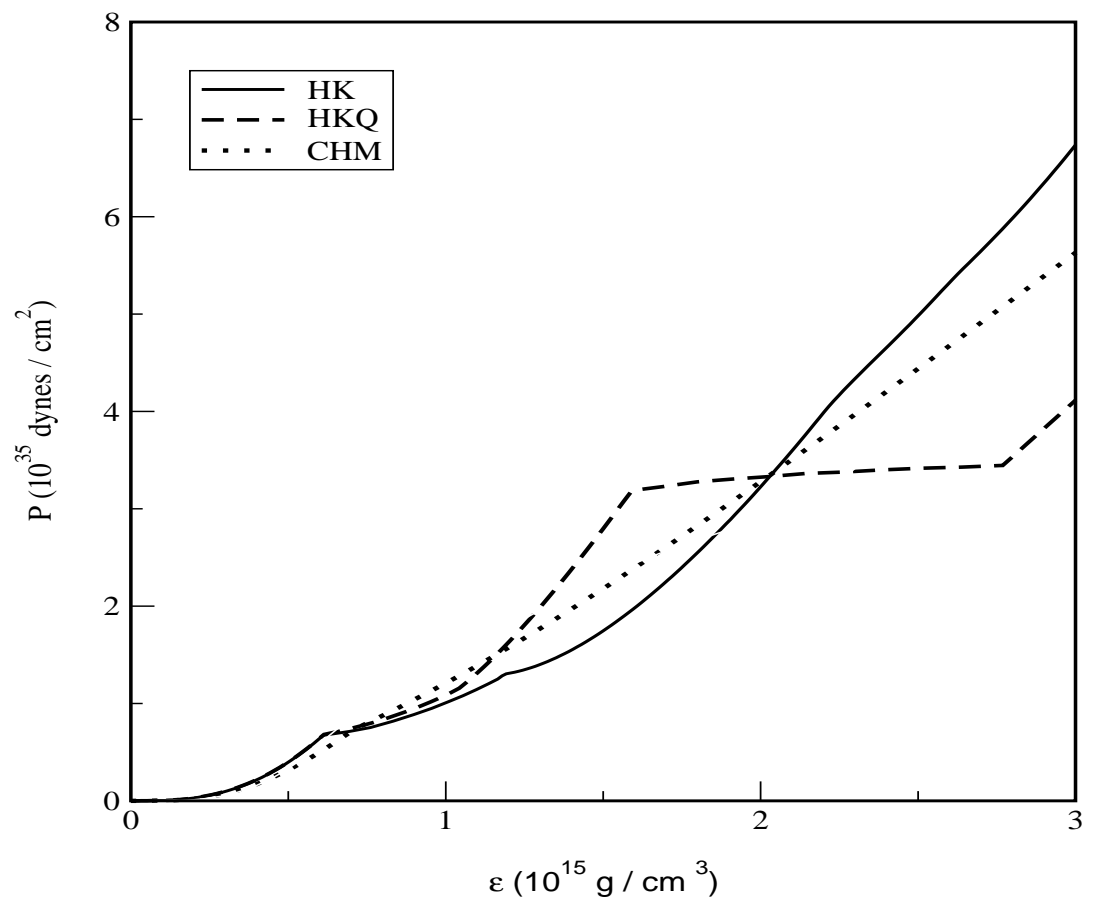

FIG. 1. The equation of state, pressure $P$ vs. energy density $\varepsilon$ for CHM, HK and HKQ cases. The results are for $n, p$, hyperon and lepton matter (dotted line), phase transitions from hadronic matter to $K^{-}$and $\bar{K}^{0}$ condensed matter (solid line) and phase transitions from nuclear matter to $K^{-}$condensed matter and then to quark matter (dashed line) calculated with antikaon optical potential depth at normal nuclear matter density of $U_{\bar{K}}=-160 \mathrm{MeV}$, bag constant $\mathrm{B}^{1 / 4}=200$ $\mathrm{MeV}$ and strange quark mass $m_{s}=150 \mathrm{MeV}$. 


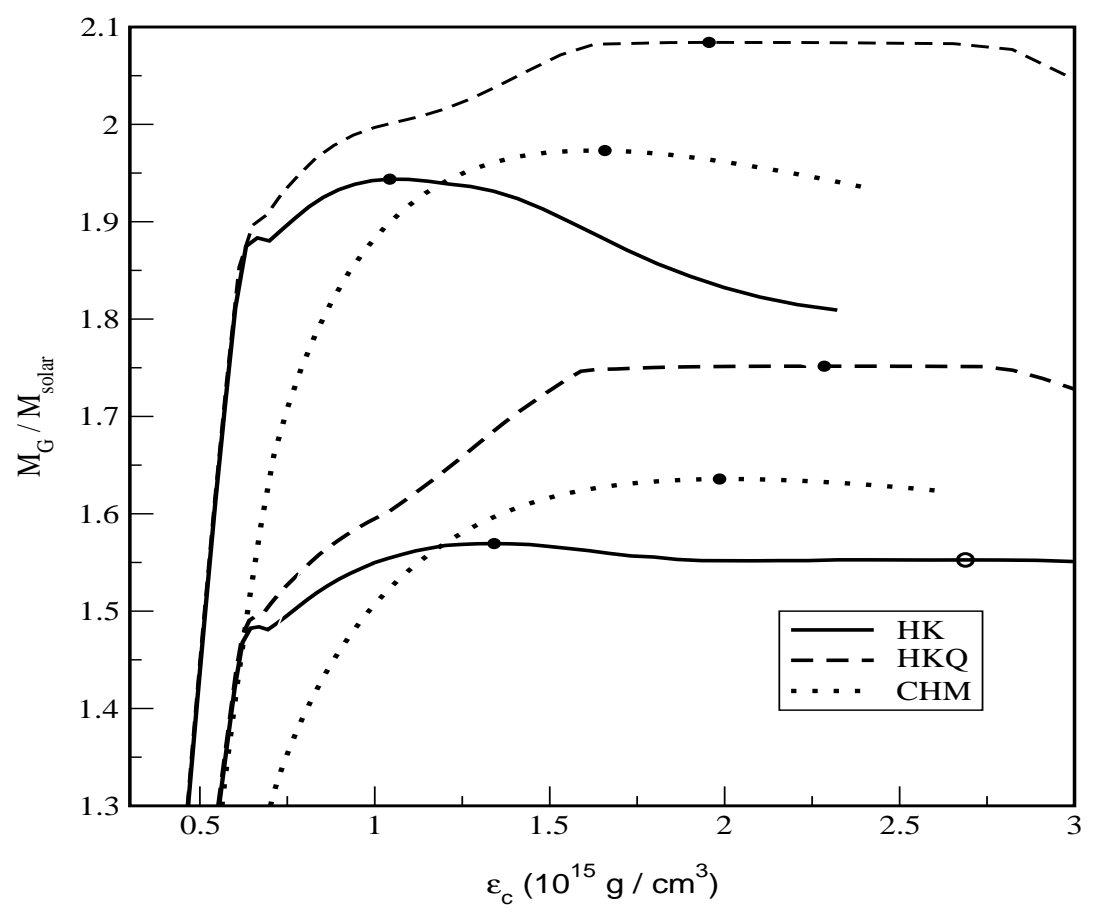

FIG. 2. The gravitational mass for static compact star sequence and mass shedding limit sequence of rotating neutron stars are plotted with central energy density for equations of state shown in FIG. 1. The different lines have the same meaning as in FIG. 1. 


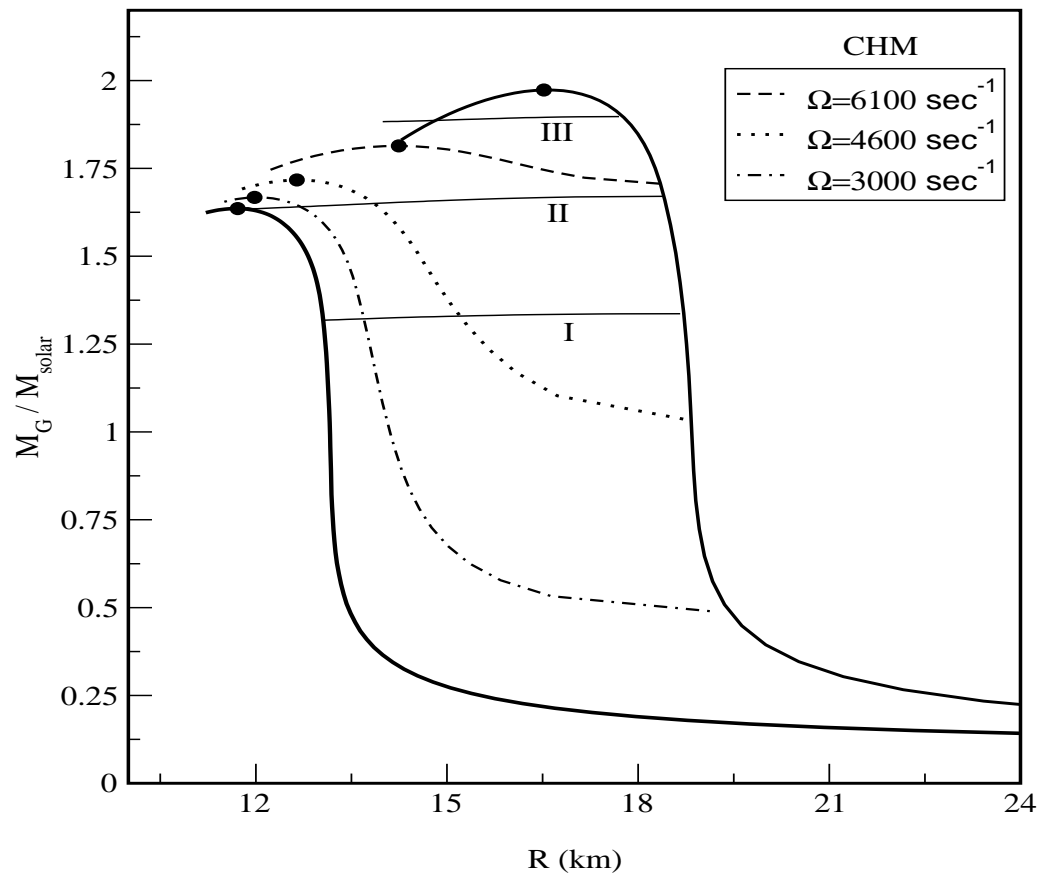

FIG. 3a. The gravitational mass as a function of equatorial radius for CHM case. The extreme left and right bold curves show the static and mass shedding limit sequence respectively. Also, fixed angular velocity $(\Omega)$ sequences are shown here. Horizontal lines are fixed baryon number sequences. Curves I and II are normal sequences and curve III is a supramassive sequence. 


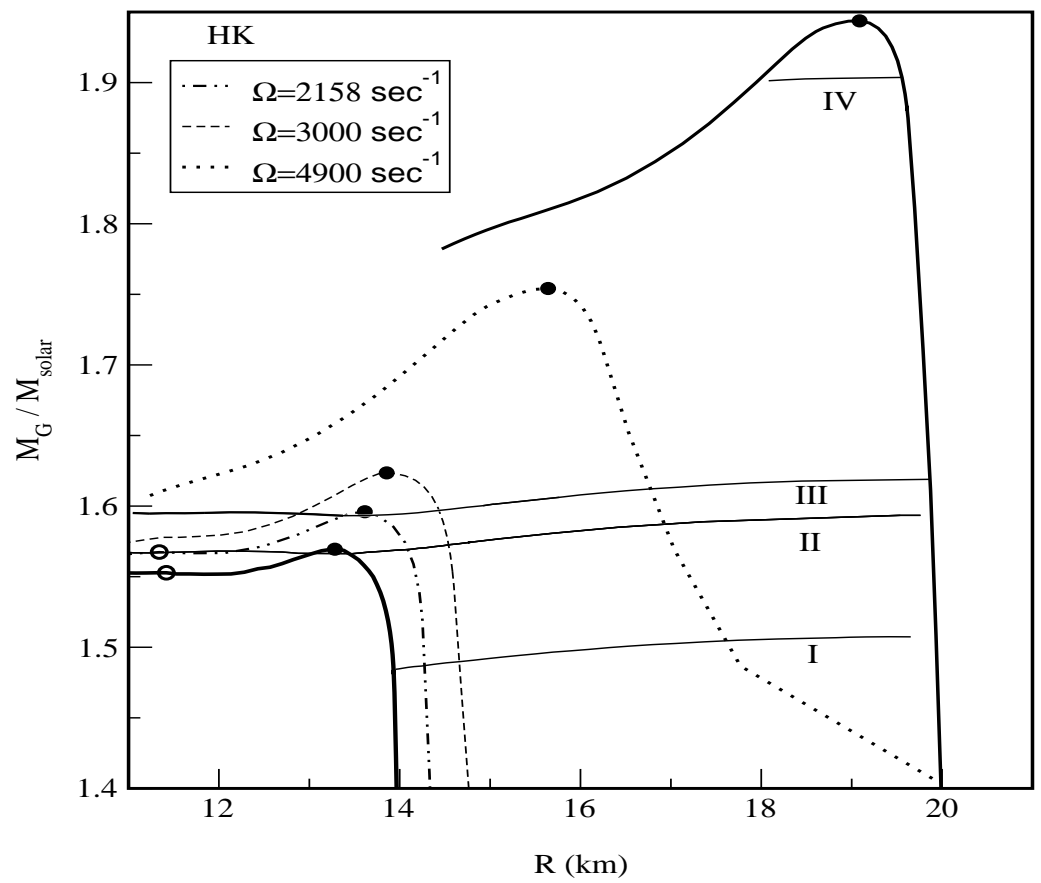

FIG. 3b. Same as FIG. 3a but for HK case. Here curves I and II are normal sequences and curves III and IV are supramassive sequences. 


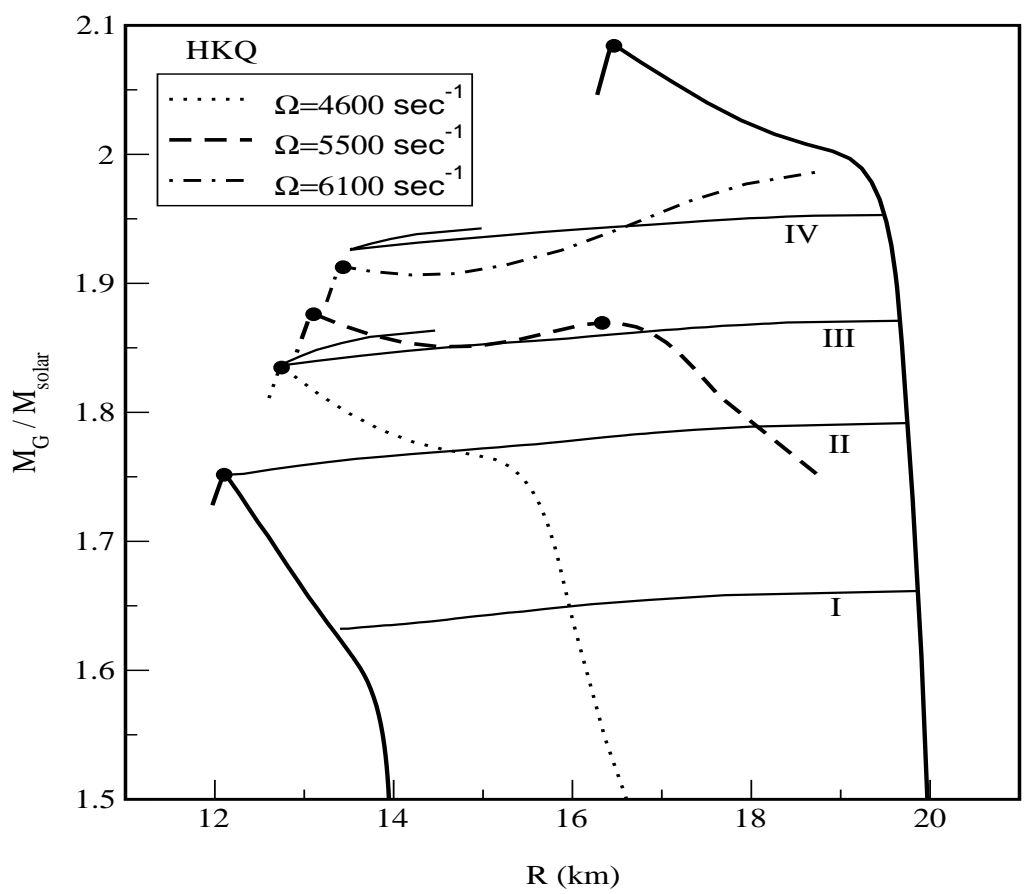

FIG. 3c. Same as FIG. 3b but for HKQ case. 


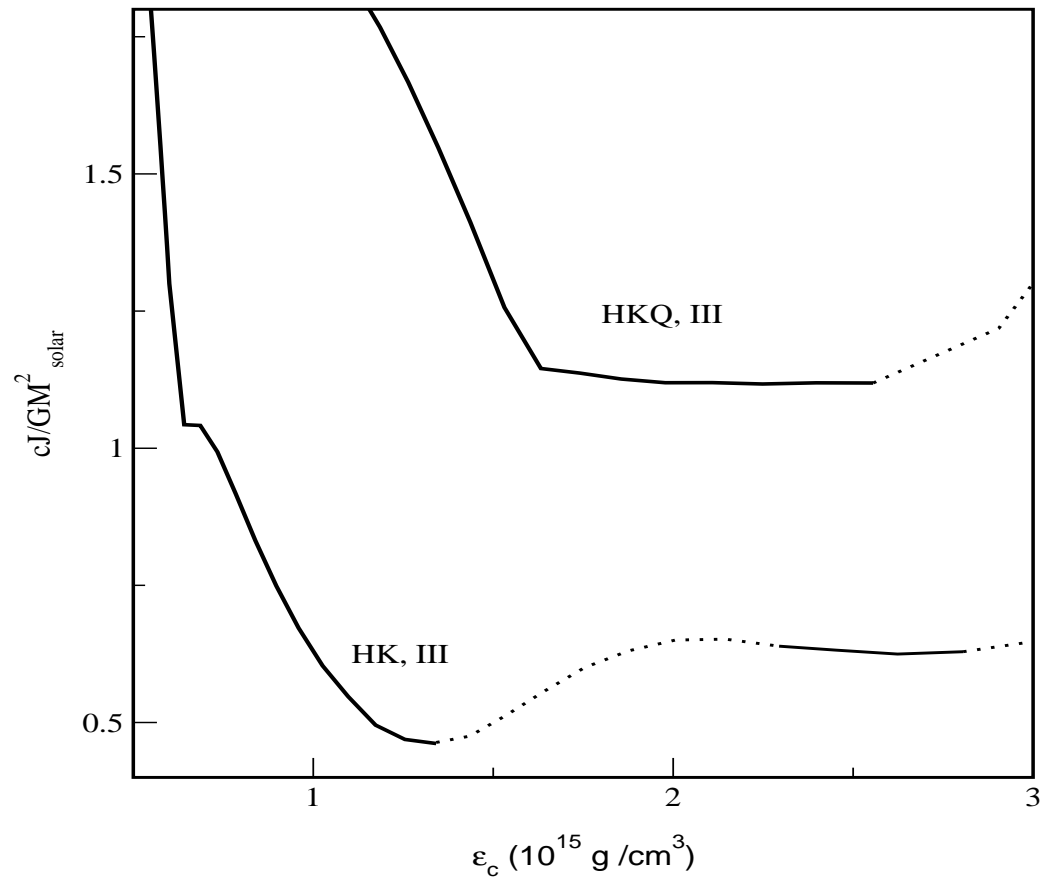

FIG. 4. The angular momentum versus central energy density for fixed baryon number supramassive sequences of HK and HKQ cases is plotted. 


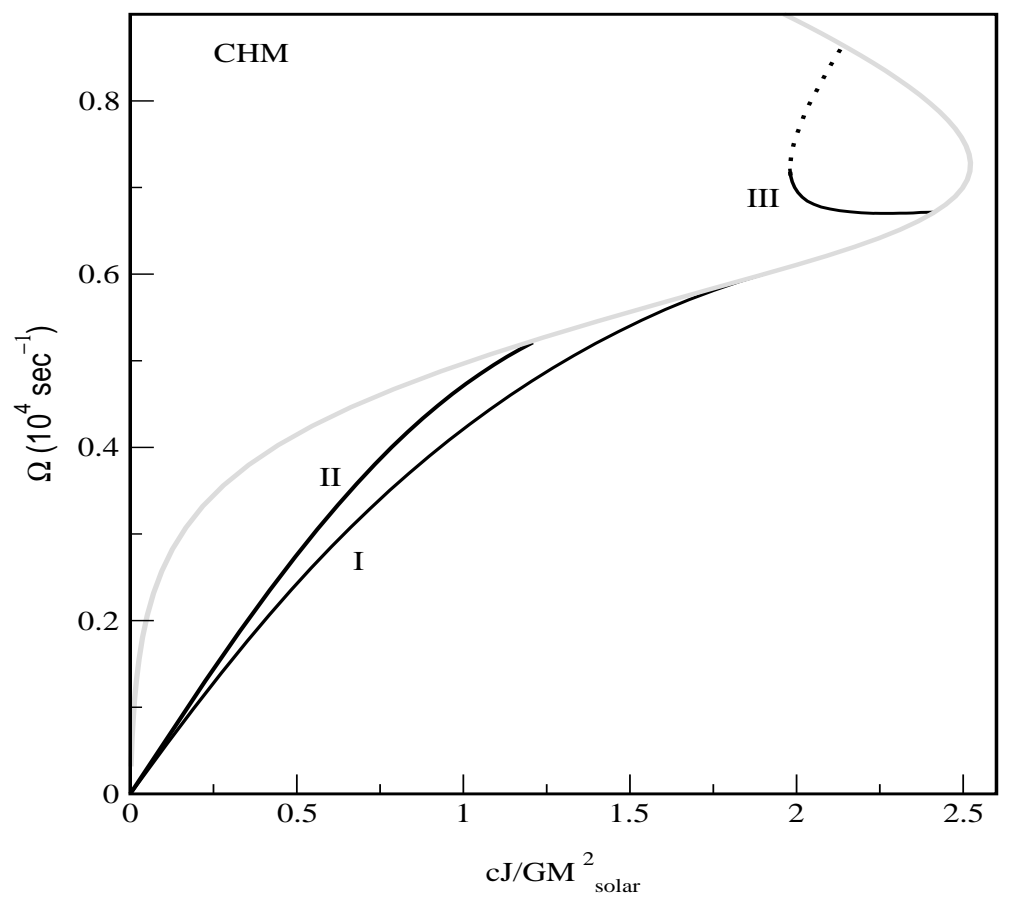

FIG. 5a. The angular velocity versus angular momentum for CHM case. All labels are as indicated in Fig. 3a. The light solid line denotes the mass shedding limit sequence. 


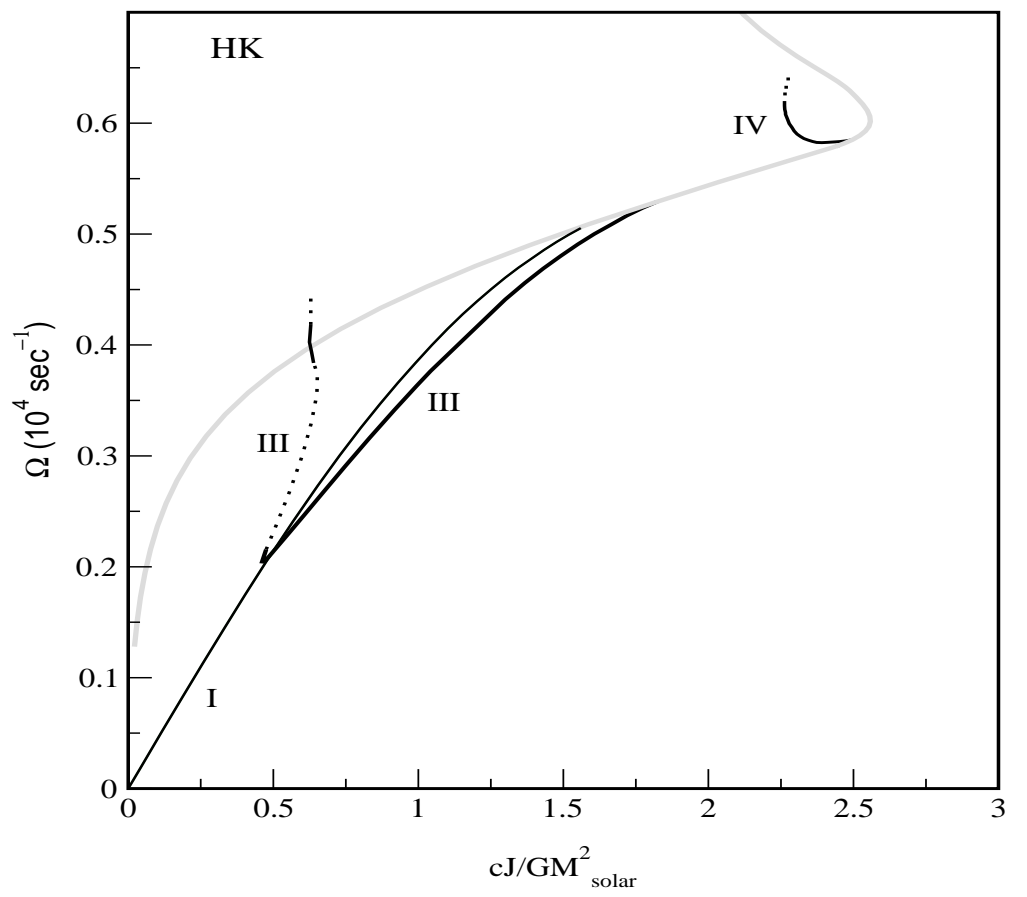

FIG. 5b. Same as FIG. 5a but for HK case. All labels are as indicated in Fig. 3b. 


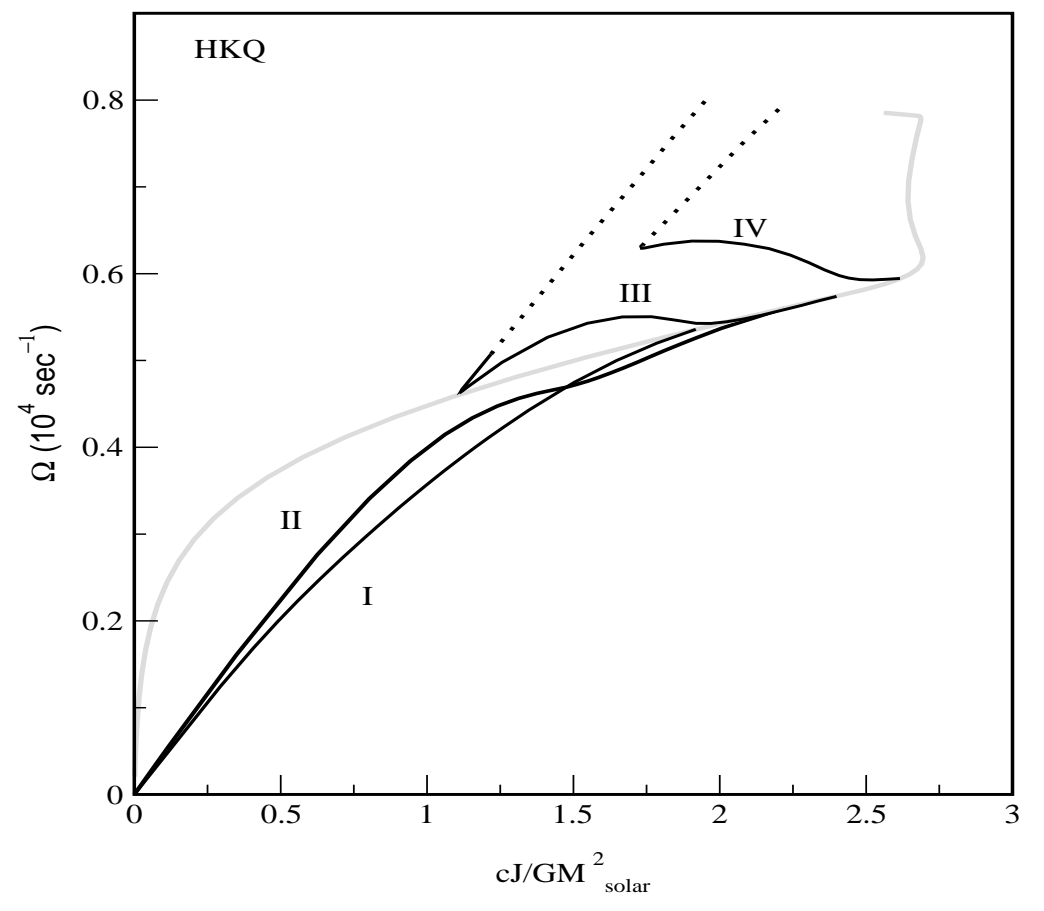

FIG. 5c. Same as FIG. 5a but for HKQ case. All labels are as indicated in Fig. 3c. 


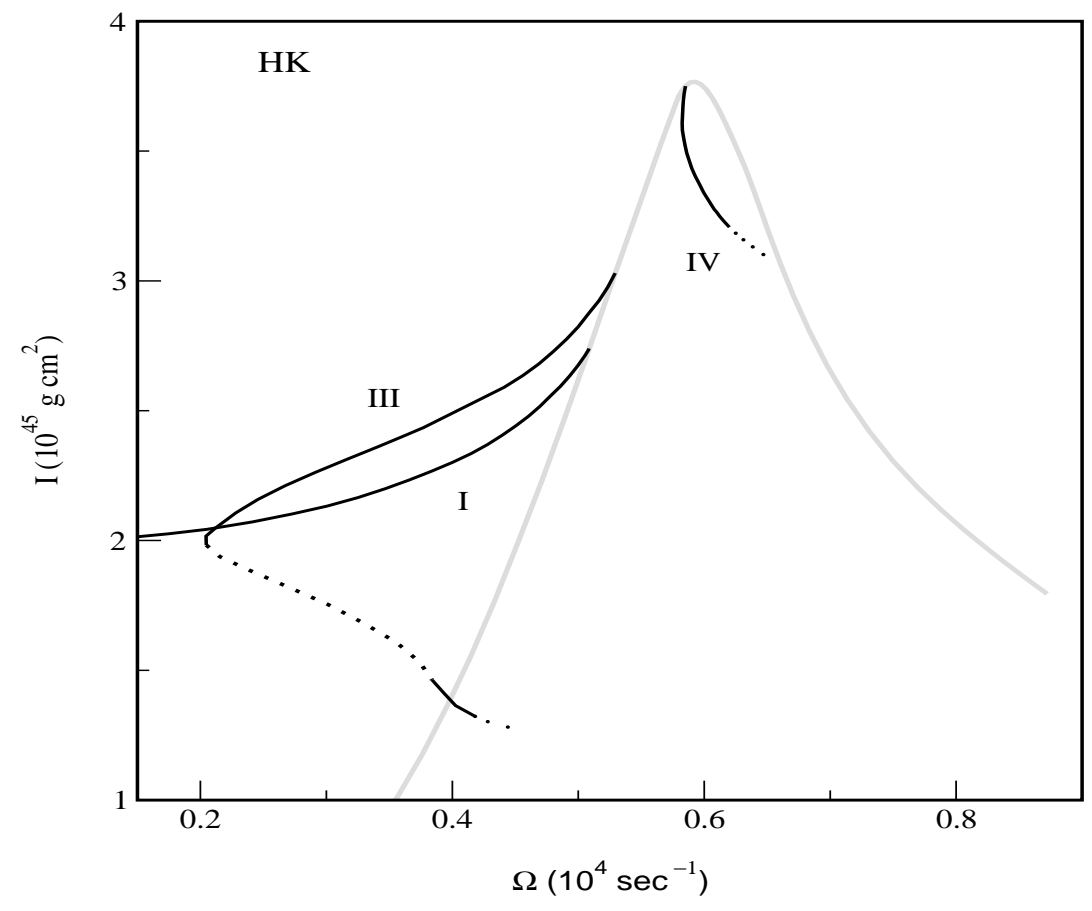

FIG. 6a. The moment of inertia versus angular velocity for HK case. All labels are as indicated in Fig. 3b. The light solid line implies the mass shedding limit. 


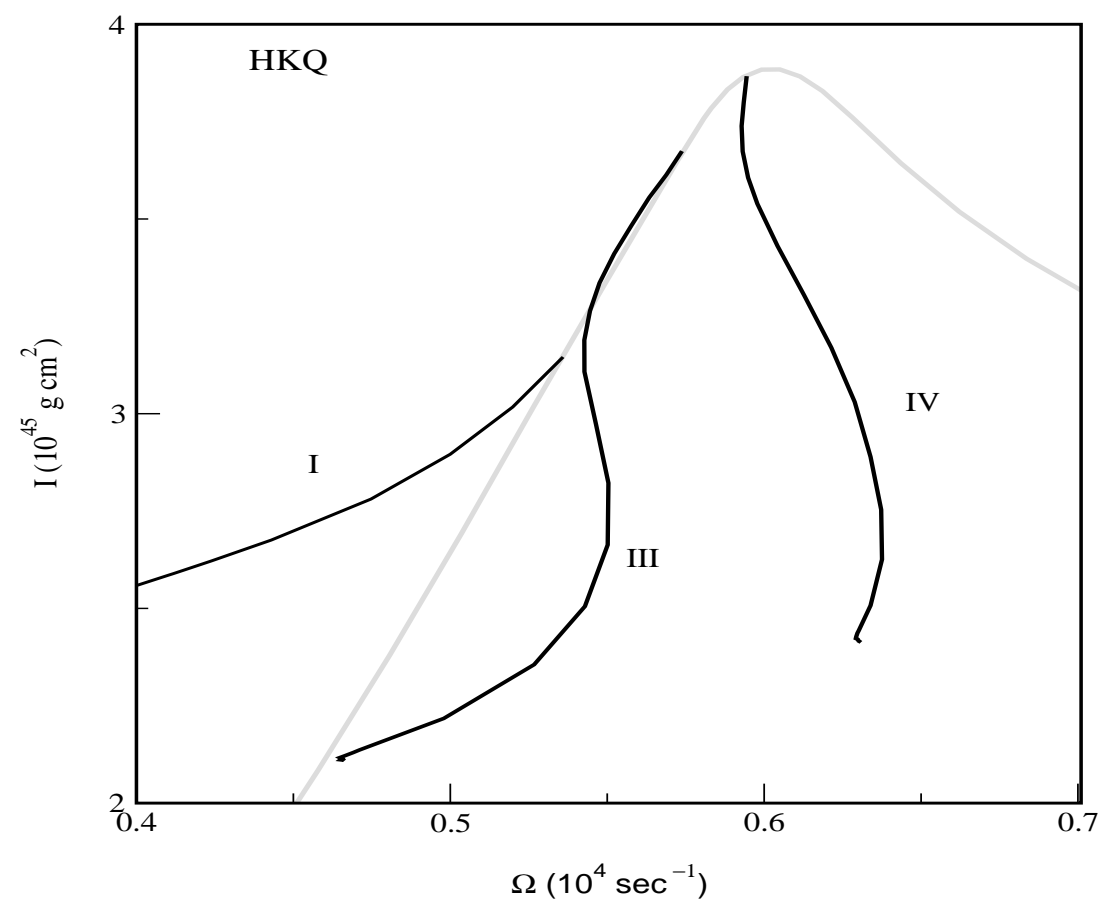

FIG. 6b. Same as FIG. 6a but for HKQ case. All labels are as indicated in Fig. 3c. 